\title{
AC 2010-1449: STUDENT-CREATED LABORATORY EXERCISES FOR A DIGITAL SYSTEMS DESIGN COURSE USING HDL AND PLDS
}

\section{Daniel McCarthy, University of Wyoming}

Daniel D. McCarthy, M.S.E.E., was a graduate student in the Department of Electrical and Computer Engineering at the University of Wyoming, Laramie, WY, from which he received both the BS in Computer Engineering and the MSEE degrees. He is a member of Tau Beta Pi and Mortar Board.

\section{Cameron Wright, University of Wyoming}

Cameron H. G. Wright, Ph.D, P.E., is an Associate Professor in the Department of Electrical and Computer Engineering at the University of Wyoming, Laramie, WY. He was formerly Professor and Deputy Department Head of the Electrical Engineerng Deparment at the U.S. Air Force Academy. His research interests include signal and image processing, real-time embedded computer systems, biomedical instrumentation, and engineering education, and is the author or co-author of over 180 publications including papers, books, and book chapters. He is a member of ASEE, IEEE, SPIE, NSPE, BMES, Tau Beta Pi, and Eta Kappa Nu; he is an active ABET evaluator and NCEES exam committee member. E-mail: c.h.g.wright@ieee.org

\section{Steven Barrett, University of Wyoming}

Steven F. Barrett, Ph.D., P.E. received the BS Electronic Engineering Technology from the University of Nebraska at Omaha in 1979, the M.E.E.E. from the University of Idaho at Moscow in 1986, and the Ph.D. from The University of Texas at Austin in 1993. He was formally an active duty faculty member at the United States Air Force Academy, Colorado and is now an Associate Professor of Electrical and Computer Engineering, University of Wyoming. He is a member of IEEE (senior) and Tau Beta Pi (chief faculty advisor). His research interests include digital and analog image processing, computer-assisted laser surgery, and embedded controller systems. He is a registered Professional Engineer in Wyoming and Colorado. He co-wrote with Dr. Daniel Pack five textbooks on microcontrollers and embedded systems. In 2004, Barrett was named "Wyoming Professor of the Year" by the Carnegie Foundation for the Advancement of Teaching and in 2008 was the recipient of the National Society of Professional Engineers (NSPE) Professional Engineers in Higher Education, Engineering Education Excellence Award.

\section{Jerry Hamann, University of Wyoming}

Jerry C. Hamann received the B.S. in Electrical Engineering with a Bioengineering Option from the University of Wyoming in 1984. He then worked for the Loveland Instrument Division of Hewlett-Packard before returning to the University of Wyoming to complete the M.S. in Electrical Engineering in 1988. Sharing time as a lecturer and National Science Foundation Graduate Fellow, he completed the Ph.D. in Electrical Engineering at the University of Wisconsin in 1993. As a faculty member at the University of Wyoming since 1993, Jerry has pursued research interests in applied robotics and control, signal processing, and higher education teaching and learning. He directed the University of Wyoming Hewlett Foundation Engineering Schools of the West Initiative until 2008, which is focused upon enhancing the recruitment, retention and quality of undergraduate engineering students. He now serves as Head, Department of Computer Science. 


\title{
Student-Created Laboratory Exercises for the Digital Systems Design Course Using HDL and PLDs
}

\begin{abstract}
The concepts presented in an introductory digital systems design lecture are often difficult for students to comprehend fully. In order to aid in this understanding, laboratory exercises are often assigned in order to reinforce the concepts introduced in lecture. These lab exercises also expose students to hardware, software, and hardware description languages used by industry professionals. We have been experimenting with a new paradigm for lab exercise creation, whereby previous students of a course are recruited to create new lab exercises for the course, a method we call "By Students, For Students" that we have tested with several different courses. This paper describes the result of applying this paradigm to a four semester hour introductory digital systems design course typically taken by sophomore electrical engineering and computer engineering majors. The lab exercises involve considerable use of programmable logic and the Verilog hardware description language (HDL). Interestingly, the student-created lab exercises tended to be more challenging than the previous set of faculty-created lab exercises.
\end{abstract}

\section{Introduction}

The concepts presented in a digital systems design lecture may be difficult for some undergraduate students to fully grasp. In order to aid in students' understanding, laboratory exercises are often used in conjunction with traditional lectures to present tough concepts in an electrical engineering courses like digital systems design. ${ }^{1-5}$

We have been experimenting with a new paradigm for lab exercise creation, whereby previous students of a course are recruited to create new lab exercises for the course, a method we call "By Students, For Students" that we have tested with several different courses. This paper describes the lab exercises that resulted from applying this paradigm to a four semester hour introductory digital systems design course (EE2390) typically taken by sophomore electrical engineering and computer engineering majors. The course includes hands-on use of Xilinx's professional-grade electronic design automation (EDA) software, Xilinx CPLDs, Atmel PLDs, and even one lab exercise using discrete logic chips. Most lab exercises involve considerable use of the Verilog hardware description language (HDL). Interestingly, the student-created lab exercises for EE2390 tended to be more challenging than the previous set of faculty-created lab exercises. Feedback from graduate student Teaching Assistants (TAs) who taught both versions of the lab exercises confirms the efficacy of the student-created content. 
EE2390 is a four credit hour course offered during the Spring and Fall semesters at the University of Wyoming. It has a prerequisite of Calculus II and is designed to be taken during a student's sophomore year. The course is taught using the fourth edition of Digital Design by M. M. Mano and M. D. Ciletti. ${ }^{6}$ The core topics covered in this course are:

- binary logic,

- digital logic gates,

- reduction of Boolean expressions,

- combinational logic design,

- SSI, MSI, LSI combinational logic ICs,

- flip-flops,

- synchronous and asynchronous sequential systems,

- MSI and LSI sequential system ICs, and

- algorithmic state machines.

The lab exercises for EE2390 are used to supplement the various concepts covered in lecture. Throughout the semester, many of the core topics covered in lecture are also covered in lab to improve the students' comprehension. However, improved understanding of lecture concepts is not the only purpose for the lab exercises in EE2390. These lab exercises also expose students to hardware, software and hardware description languages used by industry professionals. ${ }^{6-8}$ Some of these include:

- Verilog hardware description language,

- Xilinx electronic design automation (EDA) software,

- Xilinx 95108 CPLD,

- CUPL hardware description language,

- Atmel WinCUPL software, and

- Atmel ATF22V10 PLD.

The primary reason for creating a new set of lab exercises for EE2390 was to "push" the students to a higher level of understanding. The previous version of the lab exercises was created when a previous edition of the text book was used; in that edition the coverage of Verilog was not as extensive nor were there as many examples as in the latest edition. With the new textbook edition and a more thorough coverage of using HDL with programmable logic in class came the opportunity to challenge the students a bit more. However, a student's perspective about how much is too much of a challenge can be invaluable. This is part of the benefit of the "By Students, For Students" method. Previous students of some course, who are usually by now graduate students, are recruited to create new lab exercises for that course using their student perspective as a form of insight that many professors have long forgotten. We have successfully used this approach with several undergraduate courses. 


\section{Laboratory Setup}

Each lab section for EE2390 takes place in the digital lab at the University of Wyoming. Students attend one two hour lab section per week for 13 weeks out of the semester. When completing lab exercises, students work in groups of two. Although the students are working collaboratively to finish the in-lab portion of the lab exercises, each student is responsible for his/her own work and their own lab notebook.

\subsection{Laboratory Equipment}

The digital lab at the University of Wyoming is equipped with all the necessary lab equipment, hardware, and software for students to complete the assigned lab exercises. This includes:

- PCs running the Windows operating system,

- Elenco Model XK-550 (prototype board) Trainers,

- logic analyzers,

- oscilloscopes,

- multimeters,

- external power supplies,

- Xilinx’s 95108 CPLDs on custom PCBs,

- Atmel's ATF22V10CQZ PLDs in DIP packaging,

- individual logic gate in DIP packaging,

- seven segment LEDs,

- ten segment bar graph LEDs,

- Xilinx ISE 10.1 software,

- ModelSim XE III/Starter 6.3c software, and

- Atmel WinCupl software.

\subsection{Laboratory Manual}

At the start of the semester each student is required to purchase a lab manual for EE2390. ${ }^{9}$ This lab manual consists of:

- laboratory notebook procedures,

- pre-lab instructions for each lab,

- in-lab instructions for each lab,

- instructions on how to use all required software, 
- information on the custom PCBs that interface with the Xilinx 95108 CPLDs,

- a datasheet for the Atmel ATF22V10CQZ PLD, and

- datasheets for all individual logic gate chips.

\subsection{Laboratory Quizes}

At the beginning of every lab section, students are given a quiz over the material covered in the previous week's lab. These quizzes are only a few questions in length and designed to be completed in ten minutes or less.

\subsection{Students' Laboratory Notebook}

At the beginning of the semester, each student is required to purchase a blank lab notebook. The purpose of these notebooks is to get the students into the habit of keeping a valid lab notebook whenever they are doing applicable engineering work. The requirements for these notebooks are set forth in the beginning of the lab manual along with some generic guidelines as to what the students should be including in their notebook. The first couple of labs also instruct the students on exactly what should be placed into their lab notebooks. However, during the later labs, the student is responsible for including the proper information in the lab notebook. At the beginning of each lab, students submit these notebooks to the lab TA for grading. The TA grades the previous week's lab as well as the pre-lab exercises for the current week. In addition to the weekly grading of the notebooks, a final grade is given to each student's notebook at the end of semester by the course professor.

\section{Description of New Lab Exercises}

\subsection{Lab 1: Binary Arithmetic}

\subsubsection{Key Concepts}

The key concepts addressed in this lab are:

- converting between the binary, hexadecimal, and decimal number systems;

- addition and subtraction of unsigned and signed 2's complement numbers along with overflow detection;

- an introduction to the Xilinx and ModelSim software packages; and

- the simulation of a design using Xilinx and ModelSim software.

\subsubsection{Pre-Lab Exercises}

This pre-lab requires students to complete the addition and subtraction exercises shown in Table 1. Students must use the binary number system in order to complete the operations and then convert 
the result back to its hexadecimal representation. Students are also given the task of identifying when overflow occurred given that the result is going to be stored using eight bits. Finally, students check their work by converting the given numbers to their decimal representation and verifying that the results they obtained were in fact correct (if there was no overflow).

Table 1: Arithmetic Operations

\begin{tabular}{|c|c|c|c|c|c|c|c|}
\hline \multicolumn{4}{|c|}{ Unsigned Numbers } & \multicolumn{4}{|c|}{ Signed (2's Complement) Numbers } \\
\hline (1) & $8^{\prime} \mathrm{h} 23$ & $+8^{\prime} \mathrm{hCF}$ & $=$ & (6) & $8^{\prime} \mathrm{hDD}$ & $+8^{\prime} \mathrm{hEE}$ & $=?$ \\
\hline (2) & $8^{\prime} \mathrm{hA} 2$ & $+8^{\prime} h 61$ & $=$ & (7) & $8^{\prime} \mathrm{h} 71$ & $+8^{\prime} h 54$ & $=$ \\
\hline (3) & $8^{\prime}$ h 81 & $+8^{\prime} h 7 D$ & $=$ & (8) & $8^{\prime} h 9 E$ & $+8^{\prime} h A C$ & $=$ \\
\hline (4) & $8^{\prime} \mathrm{hCO}$ & $-8^{\prime} \mathrm{hAF}$ & $=$ & (9) & $8^{\prime} \mathrm{h} 4 \mathrm{E}$ & $-8^{\prime} h 1 B$ & $=$ \\
\hline (5) & $8^{\prime} h 59$ & $-8^{\prime} \mathrm{h} 74$ & $=$ & $(10)$ & $8^{\prime} \mathrm{h} 8 \mathrm{~A}$ & $-8^{\prime} h 5 B$ & $=$ \\
\hline
\end{tabular}

\subsubsection{In-Lab Exercises}

The in-lab portion of this lab is very short and does not require much work by the student. The reason for this is that during this first lab they are required to map appropriate network drives, map the network printer, and become familiar with the layout of the Xilinx software. Instructions for completing these tasks are described in detail for the students in the lab manual.

After completing all of initial lab setup, students simulate an eight bit adder subtractor schematic using the Xilinx and ModelSim software. The schematic has already been entered into a Xilinx schematic for them. A test fixture that tests the arithmetic operations that they used in the pre-lab has also already been written. Students are only required to verify that the results returned by the simulation are equivalent (including overflow detection) to the results they obtained in their pre-lab.

\subsection{Lab 2: Introduction to Combinational Logic}

\subsubsection{Key Concepts}

The key concepts addressed in this lab are:

- single bit parity generation and checking,

- DeMorgan's theorem,

- the simulation of a design using Xilinx and ModelSim software, and

- breadboarding using small IC packages of single logic gates. 


\subsubsection{Pre-Lab Exercises}

The first pre-lab exercise for this lab requires students to generate odd parity for a given set of three bit words. Then, after examining three equivalent circuits that verify odd parity for a three bit codeword and a single bit of parity, students must convert one of those circuits (shown in Figure 1) into a circuit that utilizes only NAND and inverter gates. Finally, students must provide the pin-out for the circuit that they just converted using 74LS00 and 74LS04 chips in type N packaging.

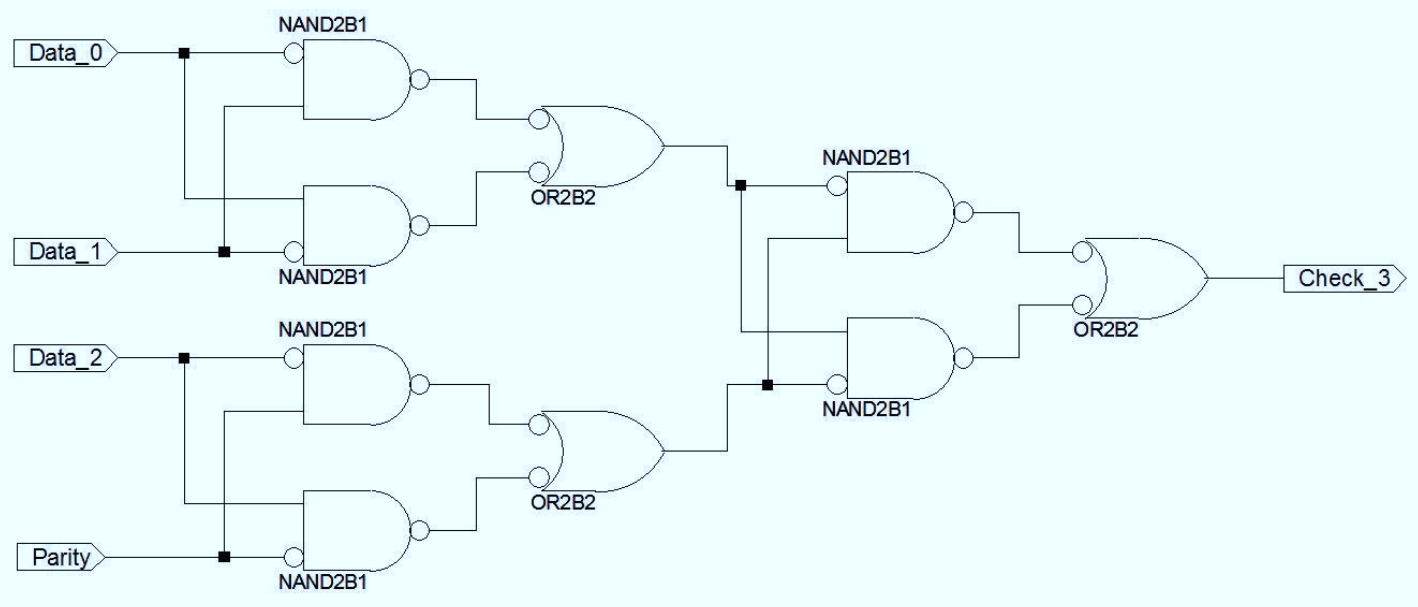

Figure 1: Odd Parity Error Detection Circuit

\subsubsection{In-Lab Exercises}

The first part of the in-lab portion of this lab requires students to simulate a completed Xilinx design. The schematic in this design contains the circuit that the students converted in the pre-lab along with two of the three other identical circuits presented earlier in the lab. A completed test fixture is also provided for the students in order to complete the simulation.

After successfully simulating their design, students are required to wire up their design on a breadboard using 74LS00 and 74LS04 chips and the pin-outs that they provided in the pre-lab. This circuit is then tested by the student to verify their design.

\subsection{Lab 3: Introduction to Combinational Logic Design and CPLD Implementation}

\subsubsection{Key Concepts}

The key concepts addressed in this lab are:

- excess-3 codes;

- an introduction to schematic design entry into the Xilinx software;

- SOP and POS expressions; 
- simplifying boolean expressions using Karnaugh maps (optional); and

- the entry, simulation, and hardware implementation of a design using Xilinx and ModelSim software.

\subsubsection{Pre-Lab Exercises}

Given a list of valid excess- 3 codes, the student's first task is to write a truth table for a signal that indicates an invalid codeword. Students must then write the canonical sum-of-products (SOP) and product-of-sums (POS) expressions for their error signal. Optionally, students can also attempt to reduce either of these expressions using Karnaugh maps. Finally, students must sketch circuit diagrams for their SOP and POS expressions.

\subsubsection{In-Lab Exercises}

For this lab, a Xilinx skeleton project is provided to the students. This skeleton project contains an unfinished schematic with only inputs and outputs drawn, a completed test fixture, and correct pin assignments for the 95108 CPLD. The first in-lab task is for the students to enter either their SOP or POS circuits into the Xilinx schematic editor. Since this is the first time that the students will actually use the schematic editor, a short introduction to this portion of the Xilinx software is provided. After successfully entering their schematic, students must simulate their design using the provided test fixture. Finally, they synthesize their design and download it to a CPLD where they can test their design in hardware.

\subsection{Lab 4: Multiple Output Combinational Logic Design with PLD Implementation}

\subsubsection{Key Concepts}

The key concepts addressed in this lab are:

- an introduction to the CUPL hardware description language,

- Gray code,

- simplifying boolean expressions using Karnaugh maps,

- the entry and hardware implementation of a design using Atmel's WinCUPL software.

\subsubsection{Pre-Lab Exercises}

The first task for the pre-lab is to complete a truth table which converts a BCD codeword to a Gray code codeword. Students then have to reduce each of the output expressions (one for each bit of the Gray code) using Karnaugh maps. After a brief introduction to CUPL, students are also tasked with writing two CUPL source files that describe the BCD to Gray code converter. One of the source files uses reduced Boolean expressions and the other uses a truth table approach. It should 
also be noted that sample CUPL files are provided that implement the solution to Lab 3 (but not this Lab 4 exercise) using reduced Boolean expressions and the truth table approach.

The CUPL language, along with the Atmel ATF22V10 PLD, are introduced in this lab in order to show students an alternative to the larger and more expensive CPLDs and FPGAs. In some cases, the "higher end" programmable logic devices such as CPLDs and FPGAs may be needed to obtain a working design, but sometimes a smaller and cheaper solution is appropriate. This lab explains this to students and gives them some experience using the smaller and cheaper 22V10 PLD.

\subsubsection{In-Lab Exercises}

The first in-lab task for the students is to properly set up a WinCUPL project. After completing this, students copy their source code file into the project and compile it. They then load their design onto the ATF22V10CQZ PLD using a dedicated computer in the lab. This design is then tested for correct operation.

\subsection{Lab 5: Multiple Output Combinational Logic Design with Verilog Implementation}

\subsubsection{Key Concepts}

The key concepts addressed in this lab are:

- an introduction to programming paradigms of Verilog (gate level, dataflow, and behavioral);

- the use of a seven-segment LED displays;

- simplifying boolean expressions using Karnaugh maps; and

- the entry, simulation, and hardware implementation of a design using Xilinx and ModelSim software.

\subsubsection{Pre-Lab Exercises}

After being given a brief introduction to the three styles of Verilog coding (which is in addition to similar coverage in lecture), the students complete a truth table describing the active low Boolean functions for driving all the segments of a seven-segment display in the manner shown in Figure 2. The input for this truth table is a single four-bit codeword. Students are then tasked to reduce each of the segment driver expressions using Karnaugh maps. Finally, students write a Verilog module that implements their seven-segment driver logic.

\subsubsection{In-Lab Exercises}

The first thing students do when they get to lab is to enter their Verilog module into a skeleton project that has been preconfigured with a test fixture (that will test the students' seven-segment driver logic), correct pin assignments, and a top-level schematic. Students then simulate their 


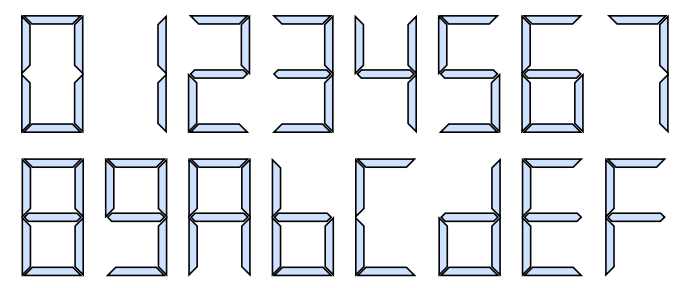

Figure 2: Desired Hexadecimal Digit Encodings for Seven-Segment Displays

design using the provided test fixture, synthesize it, and download it to the CPLD for hardware testing.

\subsection{Lab 6: Combinational Logic Design with a Decoder}

\subsubsection{Key Concepts}

The key concepts addressed in this lab are:

- incorporating previously defined logic circuits into a larger design;

- the use of a ten-segment LED bar graph; and

- the entry, simulation, and hardware implementation of a design using Xilinx and ModelSim software.

\subsubsection{Pre-Lab Exercises}

First, students are provided with the truth table for driving a ten-segment bar graph (shown in Figure 3) using a four-bit codeword. Then, students are instructed to incorporate a 3-to- 8 decoder into a combinational logic design that satisfies the truth table. Because the decoder only has eight outputs, students also have to come up with additional logic to generate the other two outputs.

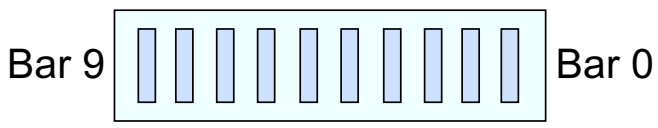

Figure 3: Ten-Segment LED Bar-Graph

\subsubsection{In-Lab Exercises}

As was the case with the previous labs, a skeleton project with a test fixture (for testing the students ten-segment bar graph), correct pin assignments, and a top-level schematic is provided for the students to use. Thus, their first task is to enter their bar graph design into the provided schematic file. Students then simulate their design using the provided test fixture, synthesize it, and download it to the CPLD for hardware testing. 


\subsection{Lab 7: Hierarchical Combinational Logic Design}

\subsubsection{Key Concepts}

The key concepts addressed in this lab are:

- hierarchical design techniques;

- the reuse of previous design modules; and

- the entry, simulation, and hardware implementation of a design using Xilinx and ModelSim software.

\subsubsection{Pre-Lab Exercises}

The design for this lab displays two numbers on seven segment displays, adds or subtracts them using unsigned addition or subtraction, and displays the result on a ten segment bar graph. Students are given the block diagram shown in Figure 4 along with a similar top-level schematic to help them visualize the hierarchial design. The pre-lab exercises for this lab require students to complete the seven-segment display module, the adder subtractor module, and the bar-graph display module using the provided skeleton modules.

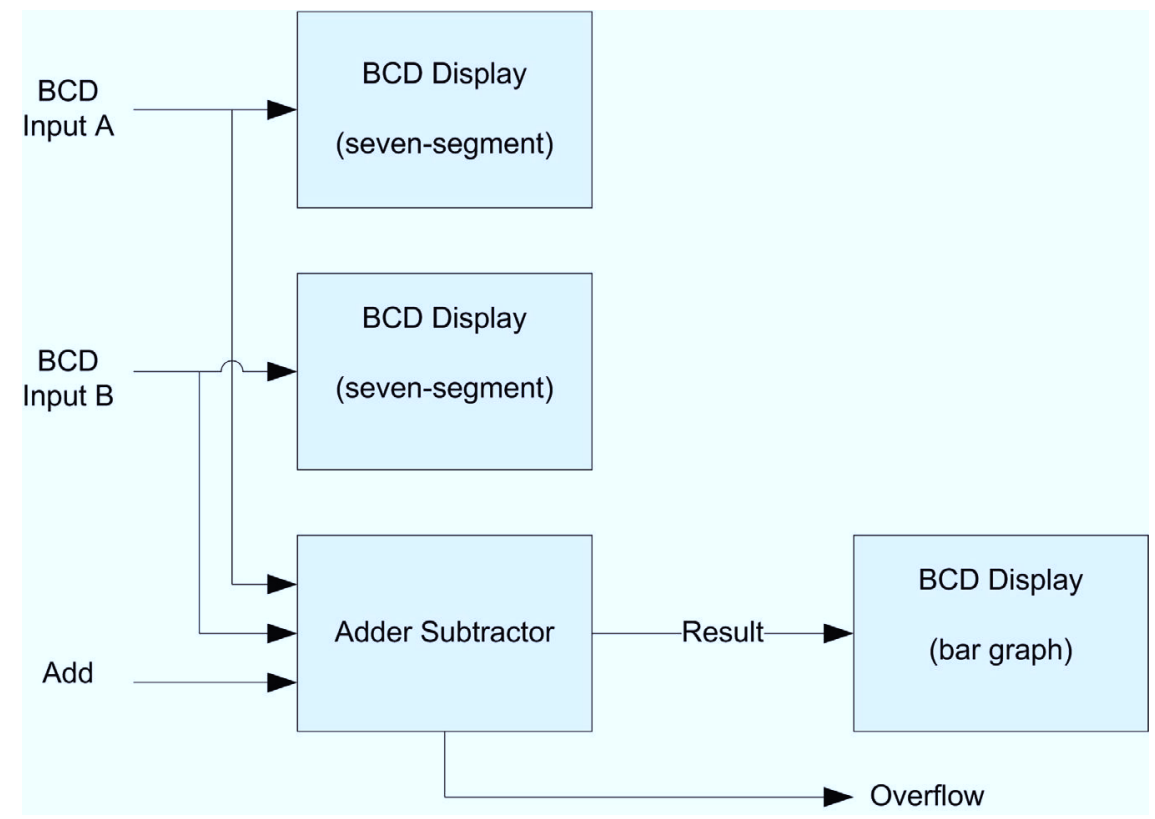

Figure 4: Block Diagram of BCD Adder

\subsubsection{In-Lab Exercises}

A skeleton project for this lab is available for the students to use when they are ready to enter their design. This skeleton project contains the skeleton Verilog source files and schematics used in the 
pre-lab, a completed top-level schematic, completed test fixtures for all four modules (the three the student created and the given top-level module), and the correct pin assignments. After entering their design into the project, students must simulate each module of their design using the test fixtures provided. After testing all of the modules, students synthesize their design and download it to a CPLD for hardware testing.

\subsection{Lab 8: Introduction to Sequential Logic Circuit Elements}

\subsubsection{Key Concepts}

The key concepts addressed in this lab are:

- operation of D, JK, and T flip-flops;

- post-fit simulation of a design;

- full schematic entry using Xilinx software;

- behavioral and post-fit simulation using Xilinx and Modelsim software; and

- hardware implementation of a design using Xilinx software.

\subsubsection{Pre-Lab Exercises}

The pre-lab portion of this lab requires students to provide timing diagrams for a positive edge triggered D flip-flop with active high output, a positive edge triggered JK flip-flp with active high output, and a cascade of three positive edge triggered $\mathrm{T}$ flip-flops with active high outputs (the inputs to all of the T flip-flops are tied to a logic high and the clocks are tied to the output of the

previous T flip-flop). Students are provided with the inputs to these flip-flop configurations and are required to complete the resulting timing diagram.

\subsubsection{In-Lab Exercises}

A skeleton project with an empty schematic file, a test fixture, and correct pin assignments is provided to the students. Their first in-lab task is to enter each of three flip-flop configurations into the single schematic file. This is the first time that students are working without a skeleton schematic. After entering the schematic, students use the provided test fixture to do a behavioral simulation on the flip-flops as well as a post-fit simulation. After completing these two simulations, students synthesize their design and download it to a CPLD for hardware testing.

\subsection{Lab 9: Clocked Synchronous State Machine Analysis}

\subsubsection{Key Concepts}

The key concepts addressed in this lab are: 
- clocked synchronous state machine analysis;

- pseudo-noise (PN) sequence generators;

- state diagrams;

- state transition/output tables; and

- the entry, simulation, and hardware implementation of a design using Xilinx and ModelSim software.

\subsubsection{Pre-Lab Exercises}

After receiving a little bit of background on PN generators, students are required to provide a single gate that self corrects the three stage PN generator shown in Figure 5 if it should ever go into an all zero state. After adding this gate students must provide a transition output table and state diagram for the PN generator. Next, students must identify whether the outputs of the PN generator in Figure 5 are Mealy or Moore outputs. Finally, students must provide a mathematical equation describing the maximum length output sequence for a PN generator with $\mathrm{n}$ different stages.

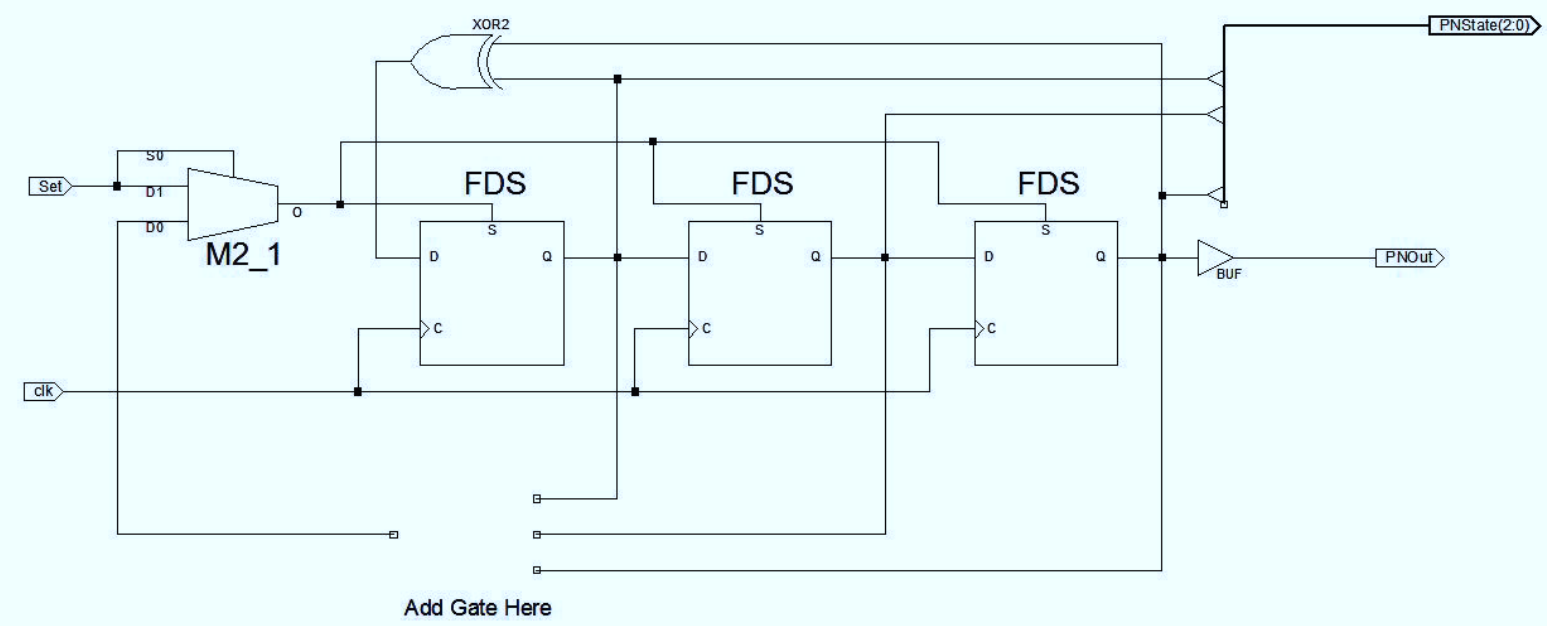

Figure 5: Three Stage PN Generator Schematic

\subsubsection{In-Lab Exercises}

A skeleton project with the schematic shown in Figure 5, a LED driver module that allows the outputs of the schematic to be displayed on a seven segment display and one segment of the bar graph, a top-level module, a test fixture, and the correct pin assignments are provided for the students. This is the last time that a skeleton project will be provided. After this lab students will be responsible for creating their own projects. The first in-lab task is for the students to complete the schematic of the PN generator using the gate they came up with in the pre-lab. After completing the schematic students simulate their design using the provided test fixture. This is also the final 
time that a test fixture will be provided for the students. After simulation is complete students synthesize their design and download it to a CPLD for hardware testing.

\subsection{Lab 10: Synchronous State Machine Design and Simulation}

\subsubsection{Key Concepts}

The key concepts addressed in this lab are:

- synchronous state machine design;

- test fixture design;

- state diagrams;

- state transition/output tables; and

- the design, entry, simulation, and hardware implementation of a design using Xilinx and ModelSim software.

\subsubsection{Pre-Lab Exercises}

This lab requires students to complete a design similar to one that was presented in class. This design was for a vending machine that accepted dimes and nickels, dispensed a $\$ 0.15$ product, and dispensed change when appropriate. In the lecture portion of the class, this design was completed using a one-hot state machine design. The design that is required for this lab is exactly the same as the one described in class except that it uses binary coded states instead of one-hot states.

The first pre-lab exercise is for the students to create a transition/output table along with a state diagram for this design. The next task is to complete a Verilog module for the vending machine. Then, students must complete a LED driver module which displays the current state of the vending machine (on a seven-segment display) and whether or not the product and or change was dispensed (using two of the ten bars on the ten-segment bar graph). Finally, students must complete a test fixture to test their vending machine module. Students may also optionally write a test fixture to test their LED driver circuit. Very sparse skeleton code files are provided to help the students in these tasks.

\subsubsection{In-Lab Exercises}

This is the first lab in which students are not given any skeleton project. This means that students will first have to create a new Xilinx project in which to enter their design. After creating this new project, students add their three completed files to the project. Then, they create a top-level module that connects the LED driver module to the vending machine module. Once this has been completed students simulate their design using their own test fixtures. If the design simulates correctly, students synthesize their design and download it to a CPLD for hardware testing using the pin assignments described in the lab manual. 


\subsection{Lab 11: Clocked Synchronous State Machine Design}

\subsubsection{Key Concepts}

The key concepts addressed in this lab are:

- synchronous state machine design;

- open ended design;

- ASM and ASMD charts;

- state transition tables; and

- the design, entry, simulation, and hardware implementation of a design using Xilinx and ModelSim software.

\subsubsection{Pre-Lab Exercises}

In this lab students are required to design a clocked synchronous state machine which drives a seven segment display in the fashion depicted in Figure 6. In this design, the state machine idles at zero until the Go input is asserted (with a logic 1). When this happens the state machine will either count up or down depending on the state of the Up input. The Go input is ignored in all states except for the idle state. On the other hand, the Up input is never ignored. There is also an active high asynchronous clear input that immediately takes the state machine back to the idle state.

The first pre-lab task for the students is for them to complete a state table and an ASM or ASMD chart (their choice) describing the required state machine. Next students must design the state machine as well as a LED driver circuit. Students may choose either a schematic or Verilog approach for either of these modules. Students must also provide test fixtures for the state machine, the LED driver circuit, and for a top-level module.

\subsubsection{In-Lab Exercises}

Once students arrive in lab, they create a new Xilinx project. Their state machine, called LED driver, and test fixtures are then added to this design. Students also must create a top-level schematic for this project which interfaces the state machine design to the LED driver design. Once this is done, students simulate their three design modules using the three test fixtures that they wrote in the pre-lab. If all design modules simulate correctly, students synthesize their design and download it to a CPLD for hardware testing using the pin assignments described in the lab manual.

\subsection{Lab 12: Final Project - State Machine Design for a Stoplight Controller}

\subsubsection{Key Concepts}

The key concepts addressed in this lab are: 


\begin{tabular}{|l||l|}
\hline Sequence for & \multicolumn{1}{|c|}{ Description } \\
\hline Seven Seg Display & $\begin{array}{l}\text { Idle state, displayed as } \\
\text { shown. Return here af- } \\
\text { ter ninth step as well as } \\
\text { when Clear=1 is de- } \\
\text { tected. }\end{array}$ \\
\hline & $\begin{array}{l}\text { First step after Go=1 } \\
\text { sensed; displayed as } \\
\text { shown. }\end{array}$ \\
\hline
\end{tabular}

Figure 6: Desired "light" Signal Pattern Sequence 
- synchronous state machine design;

- large open ended design;

- ASM and ASMD charts;

- state transition tables; and

- the design, entry, simulation, and hardware implementation of a design using Xilinx and ModelSim software.

\subsubsection{Pre-Lab Exercises}

As the final lab exercise for EE2390, it is designed to take two weeks to complete. There are also four acceptable levels of completion. Levels 1-4 correspond to maximum possible grades of 75\%, $85 \%, 100 \%$, and $110 \%$ respectively. Students are encouraged to start at level one and work their way up to level four, saving their work as they go. They are not required to go beyond level 1, but essentially all students do. Pre-lab exercises are not be graded at the beginning of this lab exercise, as the students are told they should by now be responsible for coming to lab fully prepared. It is up to the student to make sure that their design is documented to the given specifications.

In this lab exercise, students are required to control a simulated highway intersection depicted in Figure 7. To complete level 1, students must simply control lights $X$ and $Z$ using the provided timing characteristics. To complete level 2 , students must also add a crosswalk timer for both lights. The crosswalk is very similar to the design completed in lab 11 . To complete level 3 , students must also modify their control sequence to only turn the $\mathrm{X}$ lights green after a car has activated sensor C or sensor A. To complete level 4, students must also use sensors B and D to detect when a car has run a red light (light $\mathrm{X}$ ) on the north south side street. If this happens, camera $\mathrm{U}$ (for sensor $\mathrm{D}$ ) or camera $\mathrm{V}$ (for sensor $\mathrm{B}$ ) should be activated in order to take a picture of the licence plate of the offender. The crosswalk timers are displayed on seven-segment displays whereas all of the rest of the output signals are displayed using the ten-segment bar graph.

The actual pre-lab exercises are left to the students for this lab. ASM or ASMD charts, state transition tables, next-state equations, and Boolean expressions for combinational logic are all suggested as possible design tools. Students are also encouraged to use these tools at every level of their work. Finally, students are warned that if they expect to receive help from a TA or professor with their design, then they must have it properly documented using the tools mentioned above.

\subsubsection{In-Lab Exercises}

The in-lab portion of this lab is exactly the same as many of the other labs that students have completed throughout the semester. Students are responsible for simulating their design to verify functionality at all levels, synthesizing it, and downloading it to a CPLD where they can test it in hardware. In order to ensure that all students use the same LEDs, pin assignments are provided in the lab manual for this lab. 


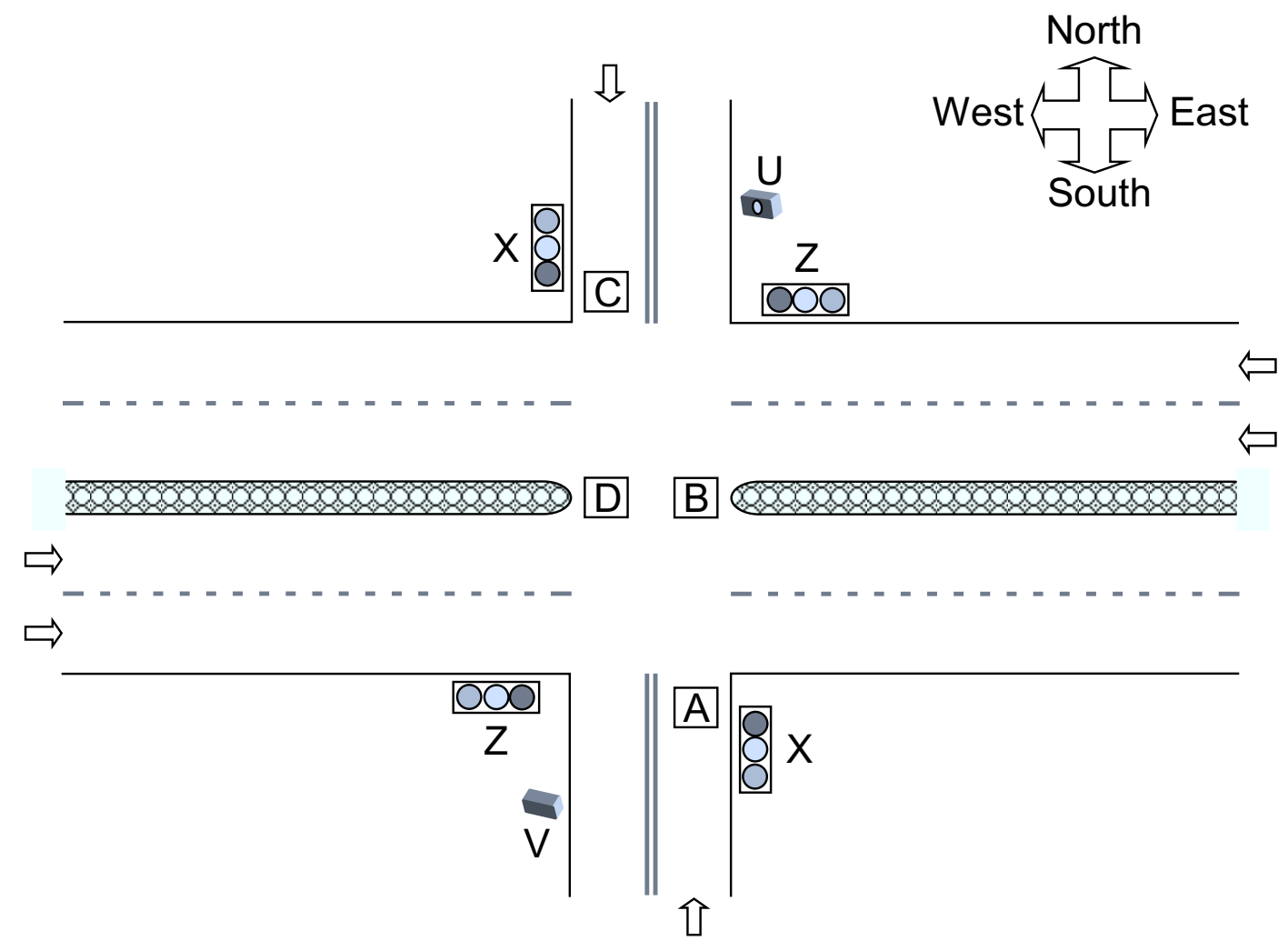

Figure 7: Intersection Used for Lab 12

\section{Conclusion}

The set of EE2390 laboratory exercises described above was created by a former EE2390 student who is now a graduate student. This is an example of our "By Students, For Students" method, which leverages a student's relatively recent memory of what what were some of the biggest hurdles for a particular course. Feedback from EE2390 TAs who taught both the previous version of the lab and this new version was interesting. Overall, the new lab exercises appeared to be "harder" and most of the individual lab grades were thus lower. However, on the final lab exercise in which the students are given an open-ended realistic design challenge, the results were much better. Thus we tentatively conclude that the more difficult lab exercises prepared the students better for open-ended design problems.

After analyzing the topic coverage of these lab exercises along with the content of related higherlevel elective courses (such as Verilog HDL and Computer Architecture) that are being offered at the University of Wyoming, we also believe that a new course in design verification techniques would be a useful addition to the curriculum. This course would teach various verification techniques, test planning, code coverage, and other similar concepts that are used by engineers in industry today.

We encourage educators to give the "By Students, For Students" method a try; we have found it to be both successful from a pedagogical standpoint but also immensely popular with students. 


\section{References}

[1] M. A. Yoder, J. H. McClellan, and R. W. Schafer, "Experiences in teaching DSP first in the ECE curriculum," in Proceedings of the 1997 ASEE Annual Conference, June 1997, paper 1220-06.

[2] R. G. Jacquot, J. C. Hamann, J. W. Pierre, and R. F. Kubichek, "Teaching digital filter design using symbolic and numeric features of MATLAB," ASEE Comput. Educ. J., vol. VII, no. 1, pp. 8-11, JanuaryMarch 1997.

[3] C. H. G. Wright and T. B. Welch, "Teaching DSP concepts using MatLaB and the TMS320C31 DSK," in Proceedings of the IEEE International Conference on Acoustics, Speech, and Signal Processing, Mar. 1999, paper 1778.

[4] J. W. Pierre, F. K. Tuffner, J. R. Anderson, D. L. Whitman, A. H. M. S. Ula, R. F. Kubichek, C. H. G. Wright, S. F. Barrett, J. J. Cupal, and J. C. Hamann, "A one-credit hour hands-on introductory course in electrical and computer engineering using a variety of topic modules," IEEE Trans. Educ., vol. 52, May 2009 , in press.

[5] S. F. Barrett, D. Whitman, R. Kubichek, J. Pierre, S. Muknahallipatna, and C. H. G. Wright, "Embedded systems design: responding to the challenge," in Proceedings of the 2009 ASEE Annual Conference, Austin, TX, June 2009.

[6] M. M. Mano and M. D. Ciletti, Digital Design, 4th ed. Prentice Hall, 2007.

[7] K. Morris, "Tilting at tech market windmills," FPGA and Programmable Logic Journal, March 2004.

[8] _ _ "Who's winning in FPGAs II," FPGA and Structured ASIC Journal, November 2008.

[9] C. H. G. Wright, J. C. Hamann, and D. D. McCarthy, EE/COSC 2390 Digital Systems Design Laboratory Manual, University of Wyoming, Laramie, WY 82071, April 2009. 